\title{
The vacuum polarization paradox and its solution
}

\author{
Engel Roza \\ Philips Research Labs, Eindhoven, The Netherlands (retired) \\ Email: engel.roza@onsbrabantnet.nl
}

\section{Summary}

In this article various heuristic approaches are discussed to solve the dark matter phenomenon by the concept of vacuum polarization. They are compared with a more fundamental approach, based upon an entropy model of the visible universe. They all make use of some kind of gravitational dipole. These dipoles seem to violate Einstein's equivalence principle between inertial mass and gravitational mass. It is shown how the paradox can be solved by a quantum mechanical principle.

Keywords: quantum gravity; vacuum polarization; gravitational dipole; Majorana particle

\section{Introduction}

In the search toward a solution of the dark matter phenomenon, various authors have proposed to consider the vacuum as a medium subject to polarization of its constituents, under influence of baryonic sources. All these proposals are struggling with the principle of equivalence between inertial mass (Newton's first law) and gravitational mass (gravitational force). In these approaches, it became clear that if there would exist some kind of a gravitational dipole, dark matter would show up as a virtual amount of matter, due to polarization of the medium that increases the gravitational field strength from a baryonic source, similarly as the electric field strength is enhanced in dielectric media. This has been shown in articles by Blanchet and Tiec [1,2], Hajdukovic [3], Raymond Penner [4] and by me [5]. The approaches, though, are different, albeit that the gravitational dipole concept is common. Its most simple configuration is a linear structure with a positive pole spaced from a negative pole with the same absolute value of its strength. Naively, the positive pole would have a strength $+m$ and the negative pole would have a strength $-m$ and the baryonic source would direct the orientation of the dipole. This would introduce the concept of negative mass and that would mean a conflict with the principle of equivalence. To escape from this problem, the various authors propose different solutions. The most simple solution is to ignore the equivalence principle and to make a distinction between gravitational mass and inertial mass. This approach has been chosen by Hajdokovic, by defining a antiparticle in his dipole that has positive inertial mass, but unlike its particle, has a negative gravitational mass. Blanchet and Tiec try to escape from the problem by making a distinction between active gravitational mass (from the source) and passive gravitational mass (from the object under acceleration). Where the passive intertial mass is equal to its gravitational mass, those of the active ones are not. Raymond Penner has chosen for an easy way out, by simply hypothesizing a new unknown particle, dubbed as virtual graviton, characterized by zero mass and a finite gravitational dipole moment. The three approaches are highly heuristic, lacking a firm theoretical basis. They are justified by fits between heuristic theory and evidence from cosmological observations. Because these fits cannot be denied, credit must be given to the concept of vacuum polarization. It is my aim in this article showing that it is possible to a give a more satisfying theoretical basis to it, by solving the vacuum polarization paradox that seems to arise from the equivalence principle.

\section{From entropy to vacuum polarization}

To this end, I would like to invoke two different, but complementary, views as outlined in a triptych of articles $[6,7,5]$. The first two of these explain the dark matter phenomenon without making use of the gravitational dipole concept. The third one relies upon some kind of gravitational dipole of a quite different nature than so far described in literature. In the first of these three articles, it has been shown that a positive value of the Cosmological Constant in Einstein's Field Equation, under the weak limit 
constraint and under particular constraints for the spatial validity range, results into a modification of Poisson's equation, such that,

$$
\nabla^{2} \Phi+\lambda^{2} \Phi=-\frac{4 \pi G M}{c^{2}} \delta^{3}(r)
$$

where $\Phi$ is the gravity potential, $G$ the gravitational constant, $c$ the vacuum light velocity, $M$ the mass of a pointlike baryonic source, $\delta(r)$ Dirac's delta function and where $\lambda$ is related with Einstein's Cosmological Constant $\Lambda$, such that

$$
\lambda^{2}=2 \Lambda=\frac{2 a_{0}}{5 M G}
$$

where $a_{0}$ is Milgrom's acceleration that characterizes the dark matter phenomenon. This view on the Cosmological Constant is different from the common perception that regards it as a constant of nature, while, like extensively motivated and analysed in [6], it is basically a integration constant in Einstein's Field Equation. It may also seem that (1) is in conflict with the commonly accepted spherical Kottler metric $[8,9,10]$ for the cosmos. The Kottler metric, however, ignores the possible existence of a uniformly distributed background energy as a bias for Einstein's Equation $[6,11,12,13]$.

The striking feature of (1) is the + sign associated with $\lambda^{2}$. If it were a - sign, the equation would be similar to Debije's equation for the potential of an electric pointlike charge in an electromagnetic plasma [14]. As is well know, the solution of such equation is a shielded Coulomb field, i.e., an electric field with an exponential decay. In the gravitational equivalent (with the + sign) the near field is enhanced ("antiscreened"), because masses are attracting, while electric charges with the same polarity are repelling. The equation holds for galaxies. Eq. (1) is, in fact, a formal expression for the Debije process that has been heuristically copied by Raymond Penner for vacuum polarization, in analogy with electromagnetism and that forced him to define his virtual graviton. However, (1) is straightforwardly been derived in [6] from Einstein's Equation. To calculate a numerical value for $a_{0}$, it appeared required to extend the analysis of a cosmological system with central mass to the visible universe with distributed matter enclosed by the Hubble time event horizon. This has been done in [7]. This has resulted into an expression for $a_{0}$ that reads as,

$a_{0}=\frac{15}{4} \Omega_{B} a_{L} ; \quad a_{L}=\frac{c}{t_{H}}$

where $t_{H}\left(\approx 13.8\right.$ Gyear) represents the Hubble time scale, and where $\Omega_{B}(=0.0486)$ is the relative share of baryonic matter in the universe, as defined and known from the Lamda-CDM model. The numerical value $a_{0} \approx 1.25 \times 10^{-10} \mathrm{~m} / \mathrm{s}^{2}$ corresponds with observational evidence.

These results (1-3) have been obtained without invoking the gravitational dipole concept. Hence the results don't suffer from the vacuum polarization paradox. However in the third triptych article, the problem pops up. In that article, Milgrom's acceleration constant has been calculated by applying the Bekenstein-Hawking entropy expression to the visible universe. This has been done by describing the vacuum as a fluid in thermodynamic equilibrium, where the molecules are subject to Heisenberg's 
energy-time uncertainty. The vibration of the molecules is modelled as the motion of an quantum mechanical oscillator with an effective mass that embodies the energy of the vibration. Note that the energy of this mass is in fact a modulation on top of the massive energy of the thermodynamic state of the fluidal molecules. To model the spin of those molecules associated with the motion, the quantum mechanical harmonic oscillator is modelled as a two-body equivalent for the purpose of assigning an dipole moment vector to the vibrating molecules. This will allow a polarization of the dipole moment vector (= directing the spin) under influences of baryonic sources. Hence, the molecules are modelled as virtual gravitational dipoles, similarly as in Hajdukovic 's theory. However, rather than a-priori identifying these virtual gravitational dipoles as pions and copying their attributes, like Hajdokovic has done, the properties of the gravitational dipoles are derived by theory. First of all, invoking the Heisenberg relationship enables the assignment of a magnitude to the dipole moment vector $\mathbf{p}_{\mathbf{d}}$ of the vibrating molecules, such that

$\left|\mathbf{p}_{\mathbf{d}}\right|=\frac{\hbar}{2 c}$

where $\hbar$ is the reduced Planck constant.

The dipole moment density $P_{g}$ amounts to

$P_{g}=\frac{a_{0}}{20 \pi G}$

Hence, from (3), (4) and (5),

$$
N \Omega_{B} p_{d}=P_{g} V \rightarrow \frac{N}{V}=\frac{a_{0}}{20 \pi G \Omega_{B}} \frac{2 c}{\hbar}=\frac{3}{8} \frac{a_{L} c}{\pi G \hbar}
$$

The total number of spins of the $N$ particles in the volume $V$ determine the information content (= entropy) in the volume $V$, which can be calculated from (6). It is proven in [5] that, by taking $V$ as the volume enclosed by the event horizon of the visible universe, and equating the result with the Bekenstein-Hawking expression, Milgrom's acceleration constant $a_{0}$ is found as in (3) indeed. The agreement between the two approaches support the viability of both.

From (6), the cell size $\Delta V$ of the vibrating gravitational molecule is found as,

$$
\Delta V=\frac{V}{N}=\frac{8}{3} \frac{\pi G \hbar}{a_{L} c}
$$

Hence, the cell radius $R$ of a spherical cell is established from,

$$
\frac{4}{3} \pi R^{3}=\frac{8}{3} \frac{\pi G \hbar}{a_{L} c} \rightarrow R=\left(\frac{2 G \hbar}{a_{L} c}\right)^{1 / 3}
$$

Inserting $G=6.6710-11 \mathrm{~m}^{3} \mathrm{~kg}^{-1} \mathrm{~s}^{-2}, \hbar=1.0510^{-34} \mathrm{~kg} \mathrm{~m}^{2} \mathrm{~s}^{-1}, a_{L}=7.110^{-10} \mathrm{~m} \mathrm{~s}^{-2}$ and $c=310^{8} \mathrm{~m} \mathrm{~s}^{-1}$, 
gives $R \approx 4.1 \mathrm{fm}$. Let us now calculate the energy of the gravitational dipole in the cell by equating its Compton wavelength with the diameter of the grain cell. Hence, from (6),

$$
h f=h \frac{1}{T}=h c \frac{1}{c T}=\frac{2 \pi \hbar c}{2 R}=\pi\left(\frac{a_{L} c}{2 G \hbar}\right)^{1 / 3}(\hbar c) .
$$

With $\hbar c=197 \mathrm{MeV} \mathrm{fm}$ and the other values just given, the gravitational dipole in would have an energy to the amount of

$\hbar \omega \approx 150 \mathrm{MeV}$.

This is pretty close to the $140 \mathrm{MeV}$ massive energy of a pion. Let $\mathrm{u}$ compare this dipole energy with the gravitational energy contained in the cell, which amounts to

$$
\Delta m c^{2}=\rho c^{2} \Delta V,
$$

where $\Delta V$ is given by (7) and where $\rho$ is the critical mass density of the flat universe, which can be found from textbooks as,

$$
\rho=\frac{3}{8 \pi G} \frac{a_{L}^{2}}{c^{2}} .
$$

Hence, from (11) and (12),

$$
\Delta m c^{2}=\frac{a_{L}}{c^{2}}(\hbar c) .
$$

This amount is extremely small and is, by far, inadequate to deliver the energy of the pion in real state. It has to be taken into account, though, that gravitational energy is not more than a tiny modulation on the energic equilibrium state of the fluidal background energy in space. The energy conflict can be avoided by taking into account that the poles in a dipole are not bound by a particle in real state, but, instead by a particle in virtual state. Considering that the energy of the gravitational dipole, calculated from theory, is close to the rest mass of a pion, Hadjuovic's heuristic assumption of virtual pions does not seem bad at all.

Now however, it may seem that we are back to the polarization paradox. That is not quite true. The gravitational dipole described in the context of the analysis by entropy is not a dipole in the sense of a construct of a particle with positive mass and an antiparticle with negative mass. Instead it is a vibrating gravitational molecule to which, by certain type of modelling, a gravitational dipole moment, is assigned. It can't be a pion, virtual or not, because the pion is a boson and the gravitational molecule is a fermion. Another issue is that, while two-body quantum mechanical oscillators can be very well modelled by a one-body equivalent, the opposite is not obvious, because it is not clear how to justify the equilibrium between an attractive force and a repelling force between the two bodies. At this point, we could choose now for the Raymond Penner solution by identifying this gravitational molecule as a hypothetical elementary particle (a virtual graviton), with a certain gravitational dipole moment as the only relevant attribute. That does not satisfy me after having shown the very basic relationships with fundamental theories as Einstein's Field Equation and the Bekenstein-Hawking entropy.

\section{Solution of the paradox}


So, let us ask ourselves what an elementary particle could be with spin (representing the gravitational dipole moment vector) that satisfies the conditions for vacuum polarization. First of all, it must be a fermion, it must be electrically neutral, it must almost stay in rest, it must have a very tiny mass only and the orientation of its spin should be subject to a baryonic gravitational field. None of the known elementary electromagnetic or nuclear particles comply with these requirements. There is, however, an exotic neutral elementary fermion that fulfils the requirements. It is a fermion, known as the Majorana particle, theorized by Ettori Majorana back in 1937 [15], so far not identified in free state. In 2012 , the particle has been identified as an electromagnetic particle in solid state by a research group headed by Leo Kouwenhoven [16]. Such a particle has a two component real valued wave function, which makes the particle state identical with its antiparticle state. Where in solid state it is the midgap state in semiconductors or in superconductors, here it is the midgap state of a molecule of an energetic fluid at thermodynamic equilibrium. This particle has, similarly as Dirac fermions, non-integer spin. In spite of its neutral charge, this spin can be influenced. In the case of electromagnetic Dirac fermions, the spin can be conceived as an elementary angular momentum. In particle physics, Dirac's theory, including Majorana's particle, not only applies to electromagnetic particles, but to all physical particles. There is no reason whatsoever why a gravitational equivalent could not exist. Hence, the vacuum polarization paradox can be solved by conceiving the vacuum particles that vibrate because of the Heisenberg uncertainty (or thermodynamic unrest), as gravitational Majorana particles. The quantum mechanical two body equivalent of a one-body quantum mechanical oscillator is just a comprehensible physical illustration how the spin of the gravitational Marjona particle is influenced by a baryonic gravitational field.

In the remainder of this article I wish to demonstrate the feasibility of the existence of an elementary physical particle with rest mass $m_{0}$, with the particular property that its momentary Einsteinean energy while moving at velocity $v_{t}$ in a gravitational potential field $\Phi$ is subject to a change, due to its spin, as,

$E_{W}^{t} \approx m_{0} c^{2}\left(1+\frac{1}{2} \frac{v_{t}^{2}}{c^{2}}\right) \rightarrow E_{W}^{t} \approx m_{0} c^{2}\left(1+\frac{1}{2} \frac{v_{t}^{2}}{c^{2}}\right)-\frac{\hbar}{2 c} \frac{1}{m_{0}} \frac{\partial}{\partial y} \Phi$.

Considering that $\Phi=m_{0} \Phi_{G}=m_{0} M G / r$, It will be clear that the additional term in this energy expression can be interpreted as the energy from a virtual gravitational dipole with a dipole moment magnitude $\hbar / 2 c$. It will appear, that such a particle not only possesses the characteristics of a Majorana particle, but that its spin has the characteristics as predicted by David Hestenes [17] for Dirac type elementary particles. He demonstrated that a Dirac particle, next to its nuclear spin (which becomes manifest as an elementary magnetic dipole moment), has an "isospin" that can be interpreted as a an elementary electric dipole moment. In the case of electromagnetic elementary particles, this electric dipole moment is insignificant, because it is dominated by the elementary magnetic dipole moment. It remains hidden in Dirac's theory, because the curl operation in common analyses is usually restricted to an operation over three dimensions, where a true space-time analysis prescribes that it should be done over four dimensions, like Hestenes did in his space-time algebra. In his article [11], Hestenes mentioned that Dirac was aware of the theoretical existence of this elementary electric moment, but that he doubted about his physical meaning [18]. Interestingly, next to Hestenes, the isospin feature has been demonstrated by Lanczos, in terms of his quaternion formalism [19], which allows a 4D equivalent for the curl operation.

It is my aim to show that, while in electromagnetism the elementary magnetic dipole moment dominates over the elementary electric dipole moment, the opposite is true for the gravitational equivalents, because, usually, the gravitational equivalent of a magnetic field is absent in gravity with pointlike baryonic sources. It will be clear that a profound analysis would require a four-dimensional 
space-time analysis. Apart from its complexity, it would bring my article out of balance. To ease the analysis, while maintaining the goal of demonstrating the existence of a hidden gravitational dipole moment in an elementary gravitational particle, $I^{\prime} l l$ do so in terms of gravitational particle moving in the direction of the field lines of a gravitational potential, which will allow a " $1+1$ " type analysis (one temporal dimension plus one spatial dimension), thereby avoiding the complexity of a full 4D-analysis. It is done in the Appendix. The end result (A-30) of the Appendix is the one shown in (14) of the main text.

\section{Conclusions}

The universe is filled with vacuum energy. The energy density $\rho c^{2}$ is given by,

$$
\rho c^{2}=\frac{c^{4}}{8 \pi G} \Lambda
$$

where $\Lambda$ is the value of Einstein's integration constant at the level of the universe.

The energy is comprised by a fluid of elementary particles in thermodynamic equilibrium. Baryonic mass in the universe is composed by local high density clusters of the elementary ones. The elementary particles are subject to quantum mechanical laws. Hence, subject to the particle/wave function duality. As a consequence, they possess spin as one of their major attributes. This spin is a virtual dipole moment with magnitude,

$$
\left|\mathbf{p}_{\mathbf{d}}\right|=\frac{\hbar}{2 c}
$$

In accordance with quantum mechanical principles, this spin is subject to quantization into two possible states. Hence, the information content or entropy of the universe is determined by the number of possible spin states of the elementary vacuum particles. The vacuum dipole moment density of the universe within the event horizon is given by

$$
P_{g}=\frac{a_{0}}{20 \pi G}
$$

where $a_{0}$ is Milgrom's acceleration constant.

Hence, the information content or entropy of the visible universe is determined by this dipole moment density, the magnitude of the spin of the elementary vacuum particles and the size of the visible universe as determined by the event horizon. The spin state of the vacuum particles is polarized by the gravitational field from the baryonic clusters.

The formal identification of elementary vacuum particles as fermionic quantum mechanical Dirac/Majorana-type ones with a spin magnitude $\hbar / 2 c$, as shown by (16) is the main result shown in this article. It confirms the more heuristic conjecture one that I derived before from the Heisenberg uncertainty [5]. The energy density formula (15) is common knowledge $[11,12,13]$. The dipole moment density expressions is the result from my previous studies $[5,6,7]$. These three main laws of vacuum energy, i.e., the energy density, the elementary vacuum particle density and the elementary spin magnitude allow the assessment of some major cosmological quantities if the size of the universe is equated with the radius of the Hubble scale event horizon and if the mass density of the universe is equated with the critical mass density that prevents the universe from collapse or explosion. As shown by (12), this critical mass density gives a value to Einstein's $\Lambda$ at the level of the visible universe. It 
does not mean, by the way, that $\Lambda$ is a constant of nature. Actually, it is an integration constant, independent of space-time coordinates, which depends on the boundary values imposed by a gravitational system under consideration. As shown by (13), the massive energy of the elementary vacuum particles is very tiny, to the amount that these are virtually mass less. This hampers a comprehensible physical interpretation of the dipole moment, such that, more or less similar to electrons, the elementary vacuum particles have to be considered as pointlike with a finite dipole moment. I am aware that the proposal to conceive the elementary fermionic gravitational particles that constitute the energic background energy in space as Majorana particles, has been put forward before, ever since 2010 [20,21,22]. It remains a challenge to relate those studies with the description given in this article.

Summarizing: the view on vacuum polarization as outlined in this article not only solves the problem how to harmonize the principle of equivalence between inertial mass and gravitational mass with the gravitational dipole concept. It also fits seamlessly with the gravitational entropic model of the visible universe inherited from the Bekenstein-Hawking entropy formula of black holes. Moreover, it gives the clue how to interpret these two different theoretical approaches for explaining the dark matter phenomenon, in terms of the quantitative empirical parameters of the Lamda-CDM model, which is considered as the Standard Model for cosmology.

\section{Appendix: The gravitational fermion}

Let us first suppose that the gravitational molecule is a free moving particle. Its Einsteinean energy is given as,

$$
E_{W}=\sqrt{\left(m_{0} c^{2}\right)^{2}+(c|\mathrm{p}|)^{2}}
$$

where $m_{0}$ is the particle's rest mass and where $\mathbf{p}$ is the threevector momentum ( $d s / d t$, not be confused with the fourvector momentum $\boldsymbol{p}$ ). Aligning the particle's free motion along the $x$-axis in Hawking metric (ict, $x, y, z), \mathrm{i}=\sqrt{-1}$ and squaring (A-1)gives,

$$
E_{W}^{2}=-p_{0}^{2}=\left(m_{0} c^{2}\right)^{2}+p_{1}^{2},
$$

which can be normalized as,

$$
p_{0}^{\prime 2}+p_{1}^{\prime 2}+1=0 ; p_{\mu}^{\prime}=\frac{p_{\mu}}{m_{0} c}
$$

Note: I prefer to use the Hawking metric (ict, $x, y, z)$ or $(+,+,+,+)$ to avoid the ugly minus sign in ($,+,+,+)$, which shows up as metric if the time dimension is defined as real instead of imaginary. As long as the temporal dimension is included, the bold italic notation for the vector $\boldsymbol{p}$ will be maintained.

Slightly differently from Dirac, Majorana wrote this equation as,

$$
p_{0}^{\prime 2}+p_{1}^{\prime 2}+1=\left(\beta+\bar{\alpha} \cdot \boldsymbol{p}^{\prime}\right)\left(\beta-\bar{\alpha} \cdot \boldsymbol{p}^{\prime}\right)=0 \text {, with } \bar{\alpha}=\bar{\alpha}\left(\alpha_{0}, \alpha_{1}\right) \text { and } \boldsymbol{p}^{\prime}\left(p_{0}^{\prime}, p_{1}^{\prime}\right) \text {, }
$$


thereby, like Dirac did, leaving freedom for the type of the number $\beta$ and for the type of components of the two-dimensional vector $\bar{\alpha}$ (Dirac wrote the equation as a full square). The elaboration of the middle term is:

$$
\begin{aligned}
& \left(\beta+\bar{\alpha} \cdot \boldsymbol{p}^{\prime}\right)\left(\beta-\bar{\alpha} \cdot \boldsymbol{p}^{\prime}\right)=\left(\beta+\sum_{\mu} \alpha_{\mu} p_{\mu}^{\prime}\right)\left(\beta-\sum_{v} \alpha_{v} p_{v}^{\prime}\right) \\
= & \beta^{2}-\beta \sum_{v} \alpha_{v} p_{v}^{\prime}+\beta \sum_{\mu} \alpha_{\mu} p_{\mu}^{\prime}-\sum_{\mu} \sum_{v} \alpha_{\mu} \alpha_{v} p_{\mu}^{\prime} p_{v}^{\prime} \\
= & \beta^{2}-\sum_{\mu \neq v}\left(\alpha_{\mu} \alpha_{v}+\alpha_{v} \alpha_{\mu}\right) p_{\mu}^{\prime} p_{v}^{\prime}-\sum_{\mu} \alpha_{\mu}^{2} p_{\mu}^{\prime 2} .
\end{aligned}
$$

To equate this middle term with the left hand term the following conditions should be true:

$\alpha_{\mu} \alpha_{v}+\alpha_{v} \alpha_{\mu}=0$ if $\mu \neq v ; \beta^{2}=1$, and $\alpha_{\mu}^{2}=-1$ for $\mu=0,1$.

From these expressions it will be clear that the numbers $\alpha_{\mu}$ and $\beta$ have to be of special type. To this end we could use, like Majorana probably did, the following matrices,

$$
\alpha_{0}=\mathrm{i} \sigma_{1}=\mathrm{i}\left[\begin{array}{ll}
0 & 1 \\
1 & 0
\end{array}\right], \quad \alpha_{1}=\mathrm{i} \sigma_{3}=\mathrm{i}\left[\begin{array}{cc}
1 & 0 \\
0 & -1
\end{array}\right], \quad \text { and } \beta=\sigma_{2}=\left[\begin{array}{cc}
0 & -\mathrm{i} \\
\mathrm{i} & 0
\end{array}\right] \text {, }
$$

where $\sigma_{i}$ are the Pauli-matrices. It can be easily verified that (A-7) meets the condition (A-6). As the momentum relationship is two-dimensional, the wave function should be two-dimensional as well. Therefore, after transforming the momenta into operators on wave functions,

$$
p_{\mu}^{\prime} \rightarrow \hat{p}_{\mu} \psi \quad \text { with } \quad \hat{p}_{\mu}^{\prime}=\frac{1}{m_{0} c} \frac{\hbar}{\mathrm{i}} \frac{\partial}{\partial x_{\mu}}
$$

(axiomatic quantum mechanical hypothesis), the momentum relationship (A-4) is transformed under consideration $(A-8)$ into the following two equivalent two-dimensional wave equations

$$
\left[\alpha_{0}\right]\left[\begin{array}{c}
\hat{p}_{0}^{\prime} \psi_{0} \\
\hat{p}_{0}^{\prime \prime} \psi_{1}
\end{array}\right]+\left[\alpha_{1}\right]\left[\begin{array}{c}
\hat{p}_{1}^{\prime} \psi_{0} \\
\hat{p}_{1}^{\prime} \psi_{1}
\end{array}\right] \pm[\beta]\left[\begin{array}{c}
\psi_{0} \\
\psi_{1}
\end{array}\right]=0, \text { and }
$$

or, for (A-9a) with explicit expressions of the Pauli matrices,

$$
\left[\begin{array}{cc}
0 & \mathrm{i} \\
\mathrm{i} & 0
\end{array}\right]\left[\begin{array}{c}
\hat{p}_{0}^{\prime} \psi_{0} \\
\hat{p}_{0}^{\prime} \psi_{1}
\end{array}\right]+\left[\begin{array}{cc}
\mathrm{i} & 0 \\
0 & -\mathrm{i}
\end{array}\right]\left[\begin{array}{c}
\hat{p}_{1}^{\prime} \psi_{0} \\
\hat{p}_{1}^{\prime} \psi_{1}
\end{array}\right] \pm\left[\begin{array}{cc}
0 & -\mathrm{i} \\
\mathrm{i} & 0
\end{array}\right]\left[\begin{array}{c}
\psi_{0} \\
\psi_{1}
\end{array}\right]=0
$$

This reads as the following two equations:

$$
\mathrm{i} p_{0}^{\prime} \psi_{1}+\mathrm{i} \hat{p}_{1}^{\prime} \psi_{0} \mp \mathrm{i} \psi_{1}=0 \text { and } \mathrm{i} \hat{p}_{0}^{\prime} \psi_{0}-\mathrm{i} \hat{p}_{1}^{\prime} \psi_{1} \pm \mathrm{i} \psi_{0}=0 \text {, }
$$

After denormalization (Eq. (8)) and written in matrix terms 


$$
\left[\begin{array}{cc}
\mathrm{i} \hat{p}_{1} & \mathrm{i} \hat{p}_{0} \mp \mathrm{i} m_{0} c \\
\mathrm{i} \hat{p}_{0} \pm \mathrm{i} m_{0} c & -\mathrm{i} \hat{p}_{1}
\end{array}\right]\left[\begin{array}{l}
\psi_{0} \\
\psi_{1}
\end{array}\right]=0 .
$$

Let the upper signed part of the two equations be heuristically solved by

$$
\psi_{i a}(x, t)=u_{i a} \operatorname{expi}\left(\frac{p_{1}}{\hbar} x-\frac{W}{\hbar} t\right)
$$

and the lower signed part by

$$
\psi_{i b}(x, t)=u_{i b} \exp -\mathrm{i}\left(\frac{p_{1}}{\hbar} x-\frac{W}{\hbar} t\right)
$$

After substitution of (A-13a) into the upper signed part of (A-12) we find

$$
\left[\begin{array}{cc}
p_{1} & \mathrm{i}\left(W / c-m_{0} c\right) \\
\mathrm{i}\left(W / c+m_{0} c\right) & -p_{1}
\end{array}\right]\left[\begin{array}{l}
u_{0} \\
u_{1}
\end{array}\right]=0 .
$$

Substitution of $(A-13 b)$ into the lower signed part of $(A-12)$ gives,

$$
\left[\begin{array}{cc}
-p_{1} & -\mathrm{i}\left(W / c+m_{0} c\right) \\
-\mathrm{i}\left(W / c-m_{0} c\right) & p_{1}
\end{array}\right]\left[\begin{array}{l}
u_{0} \\
u_{1}
\end{array}\right]=0 .
$$

Non-trivial solutions for $\psi_{i}$ are obtained if the determinant of the matrix is zero. In both cases this is true if:

$$
\left(\frac{W}{c}\right)^{2}=p_{1}^{2}+m_{0}^{2} c^{2}
$$

This condition corresponds with the square of the Einsteinean relationship (A-1). It makes (A-13a) and $(A-13 b)$ valid solutions. This marks the solution of Majorana's equation as two equivalent twocomponent solutions (A-13). The amplitude ratio of the two components is given by

$$
\frac{u_{0}}{u_{1}}=-\mathrm{i} \frac{W / c-m_{0} c}{p_{1}}=\frac{p_{1}}{\mathrm{i}\left(W / c+m_{0} c\right)} .
$$

Unlike as in the case of Dirac's approach, the expansion of of (A-4) results into two independent wave function solutions under the same conditions. The general solution is the sum of the two. Hence,

$$
\psi(x, t)=u_{i}\left\{\operatorname{expi}\left(\frac{p_{1}}{\hbar} x-\frac{W}{\hbar} t\right)+\exp -\mathrm{i}\left(\frac{p_{1}}{\hbar} x-\frac{W}{\hbar} t\right)\right\}=2 u_{i} \cos \left(\frac{p_{1}}{\hbar} x-\frac{W}{\hbar} t\right) .
$$

Unlike the wave function of a (two-component) Dirac-type particle, the wave function of a (two component) Majorana-type particle is real. The particle is its own antiparticle. 
In the non-relativistic condition, where $v<<c$, we have

$W / c= \pm \sqrt{p_{1}^{2}+m_{0}^{2} c^{2}} \approx \pm m_{0} c\left(1+\frac{1}{2} \frac{v^{2}}{c^{2}}\right)$

Under this condition, we have for positive energy $W / c>0$,

$$
\frac{u_{0}}{u_{1}} \approx-\mathrm{i} \frac{v}{2 c}
$$

Apart from the dominant component, there is a small second component, which is responsible for a particular property of the wave function. In the case of electrons such property is known as spin.

The interpretation of spin requires an extension of the analysis from the free moving condition to the condition of motion under influence of a potential field. In the following analysis we shall make use of the following identities,

$$
\begin{aligned}
& (\bar{\alpha} \cdot \mathbf{v})(\bar{\alpha} \cdot \mathbf{w})=\mathbf{v} \cdot \mathbf{w} \pm \mathrm{i}(\mathbf{v} \times \mathbf{w}) \\
& (\mathbf{v}+\mathbf{w}) \times(\mathbf{v}+\mathbf{w})=(\mathbf{v} \times \mathbf{w})+(\mathbf{w} \times \mathbf{v}) \\
& \nabla \times(\mathbf{v} \psi)=\psi(\nabla \times \mathbf{v})+\nabla \psi \times \mathbf{v}
\end{aligned}
$$

In these relationships $\mathbf{v}$ and $\mathbf{w}$ are two-dimensional vectors, the quantity $\bar{\alpha}=\bar{\alpha}\left(\alpha_{0}, \alpha_{1}\right)$ consists of components equal to the $2 \times 2$ Pauli- type matrices in (A-7) and the quantity $\psi$ is a scalar field. The first relationship is a vector identity that relies upon the properties of the Pauli-type matrices. The second identity is a property from elementary vector calculus. The third one is the expansion of the curl operation on the product of a spatial vector and a spatial scalar field.

Eq. (A-8) in terms of $(A-20)$ is equivalent with

$$
\left(\bar{\alpha} \cdot \boldsymbol{p}^{\prime}\right)\left(\bar{\alpha} \cdot \boldsymbol{p}^{\prime}\right)-\beta^{2}=\boldsymbol{p}^{\prime} \cdot \boldsymbol{p}^{\prime} \pm \mathrm{i}\left(\boldsymbol{p}^{\prime} \times \boldsymbol{p}^{\prime}\right)-\beta^{2}=0 .
$$

This might seem a trivial result, because the outer product of a vector with itself is zero. Hence, this is just a retrieval of the Einsteinean energy expression. The expression however changes under the change of momenta components as a consequence of the (normalized) vector potential $\boldsymbol{A}^{\prime}$ of a conservative field, such that

$$
\boldsymbol{p}^{\prime} \rightarrow \boldsymbol{p}^{\prime}+\boldsymbol{A}^{\prime} \text { and } E_{W}^{2} \rightarrow\left(\boldsymbol{p}^{\prime}+\boldsymbol{A}^{\prime}\right)\left(\boldsymbol{p}^{\prime}+\boldsymbol{A}^{\prime}\right) \pm\left(\boldsymbol{p}^{\prime}+\boldsymbol{A}^{\prime}\right) \times\left(\boldsymbol{p}^{\prime}+\boldsymbol{A}^{\prime}\right)-\beta^{2}
$$

The outer product in this expression still seems being irrelevant, because of its zero value. This, however, changes after the quantum mechanical transform from momenta to operations on a wave function. Applying these transforms on the identity

$\left(\boldsymbol{p}^{\prime}+\boldsymbol{A}^{\prime}\right) \times\left(\boldsymbol{p}^{\prime}+\boldsymbol{A}^{\prime}\right)=\left(\boldsymbol{p}^{\prime} \times \boldsymbol{A}^{\prime}\right)+\left(\boldsymbol{A}^{\prime} \times \boldsymbol{p}^{\prime}\right)$

we have

$$
\left(\boldsymbol{p}^{\prime} \times \boldsymbol{A}^{\prime}\right)+\left(\boldsymbol{A}^{\prime} \times \boldsymbol{p}^{\prime}\right) \rightarrow\left(\hat{\boldsymbol{p}}^{\prime} \times \boldsymbol{A}^{\prime}\right) \psi+\left(\boldsymbol{A}^{\prime} \times \hat{\boldsymbol{p}}^{\prime}\right) \psi
$$


Where the operator in the first term operates on $\psi$ as well as on $\boldsymbol{A}^{\prime}$, the operator in the second term only operates on $\psi$. As a consequence (A-23) is evaluated as,

$$
\left(\hat{\boldsymbol{p}}^{\prime} \times \boldsymbol{A}^{\prime}\right) \psi+\left(\boldsymbol{A}^{\prime} \times \hat{\boldsymbol{p}}^{\prime}\right) \psi=\frac{\hbar}{\mathrm{i}} \psi\left(\nabla \times \boldsymbol{A}^{\prime}\right) .
$$

Curiously, where the outer product of the momentum representation is zero, the equivalent wave function representation is not. Apparently, the expression of the Einsteinean energy under influence of spin changes as,

$$
\left(\boldsymbol{p}^{\prime}+\boldsymbol{A}^{\prime}\right)\left(\boldsymbol{p}^{\prime}+\boldsymbol{A}^{\prime}\right)-\beta^{2}=0 \rightarrow\left(\boldsymbol{p}^{\prime}+\boldsymbol{A}^{\prime}\right)\left(\boldsymbol{p}^{\prime}+\boldsymbol{A}^{\prime}\right)-\beta^{2}+\frac{\hbar}{\mathrm{i}}\left(\nabla \times \boldsymbol{A}^{\prime}\right)=0 .
$$

Let us proceed by expanding this equation under consideration that the potential field is generically vectorial, i.e., that next to its scalar component $\Phi$, a vector component $\mathbf{A}$ has to be taken into consideration. In spite of the particle's motion in one spatial direction, we shall suppose that the vectorial component has, next to a zero component $A_{x}$, a zero valued transversal component $A_{y}$ such that

$$
\begin{gathered}
\boldsymbol{p}^{\prime}=\boldsymbol{p}^{\prime}\left(p_{0}^{\prime}, p_{1}^{\prime}\right) ; \boldsymbol{A}^{\prime}=\boldsymbol{A}^{\prime}\left(\mathrm{i} \frac{\Phi / c}{m_{0} c}, 0,0\right) ; \beta^{2}=-1, \text { and } \\
\nabla \times \boldsymbol{A}^{\prime}=\left[\begin{array}{ccc}
\mathrm{e}_{\mathrm{t}} & \mathrm{e}_{\mathrm{x}} & \mathrm{e}_{\mathrm{y}} \\
\partial / \mathrm{i} c t & \partial / \partial x & \partial / \partial y \\
\mathrm{i} \Phi / m_{0} c^{2} & 0 & 0
\end{array}\right]=-\frac{\partial}{\partial y} \mathrm{i} \frac{\Phi}{m_{0} c^{2}} \mathrm{e}_{\mathrm{x}}
\end{gathered}
$$

where $\mathbf{e}_{\mathbf{x}}, \mathbf{e}_{\mathbf{y}}$ and $\mathbf{e}_{\mathrm{t}}$, respectively, are unit vectors along the two spatial axes and the temporal axis. and the spatial axis.

Note: The i factor in the scalar component is due to the (Hawking) metric choice $(+,+,+,+) /($ ict, $x, y, z)$. It can be easily seen from the Lorentz gauge

$$
\nabla \cdot \mathbf{A}+\frac{1}{c^{2}} \frac{\partial \Phi}{\partial t}=0 \rightarrow \nabla \cdot \mathbf{A}+\mathrm{i} \frac{\partial \Phi / c}{\partial \mathrm{i} c t}=0
$$

Note also that $\Phi / m_{0} c^{2}$ is a dimensionless quantity and that the gravitational potential is an energy per unit of mass. Hence

$$
\Phi_{G}(r)=\frac{M G}{r}=\frac{\Phi}{m_{0}}
$$

Note: it is at this point, where we move on with gravity. Moving on with a pointlike charge $q_{e}$ in electromagnetism would be possible under the condition,

$$
\Phi_{E M}(r)=\frac{q_{e}}{4 \pi \varepsilon_{0}} \frac{1}{r}
$$


Consequently, (A-25) evolves as

$$
\left(p_{0}^{\prime}+\frac{\mathrm{i} \Phi}{m_{0} c^{2}}\right)^{2}+p_{1}^{\prime 2}+\hbar \frac{\partial}{\partial y} \frac{\Phi}{m_{0} c^{2}}+1=0
$$

After denormalization

$$
\left(\frac{p_{0}}{m_{0} c}+\frac{\mathrm{i} \Phi}{m_{0} c^{2}}\right)^{2}+\left(\frac{m_{0} v}{m_{0} c}\right)^{2}+\hbar \frac{\partial}{\partial y} \frac{\Phi}{m_{0} c^{2}}+1=0
$$

where $v$ is the particle's velocity.

Note that $p_{0}$ is imaginary, as can be seen from (A-2).

Hence, under consideration of (A-2),

$$
\left(\frac{E_{W}}{m_{0} c^{2}}\right)^{2}=-\left(\frac{p_{0}}{m_{0} c}+\frac{\mathrm{i} \Phi}{m_{0} c^{2}}\right)^{2}=\left(\frac{m_{0} v}{m_{0} c}\right)^{2}-\frac{\hbar}{m_{0} c} \frac{\partial}{\partial y} \frac{\Phi}{m_{0} c^{2}}+1
$$

Supposing that the first two terms in the most right-hand part are much smaller than 1 ,

$$
E_{W} \approx\left(m_{0} c^{2}\right)\left(1+\frac{1}{2} \frac{v^{2}}{c^{2}}\right)-\frac{\hbar}{2 c} \frac{1}{m_{0}} \frac{\partial}{\partial y} \Phi
$$

The last term in the right hand part of (A-29) is the influence of a torque from a gravitational field perpendicular on the direction of motion. The dimension of the product of the particle's mass and the product of the particle's mass and patial derivative of a gravitational potential is that of a force. That makes $\hbar / 2 c$ an elementary gravitational dipole moment. Curiously, whatever the mass of the particle is this elementary dipole moment always has the same value. It is just a consequence of the particle/wave duality. Condition (A-29) is not a prerequisite, it is adopted here to reveal the physical interpretation of $\hbar / 2 c$ as a virtual dipole moment $\hbar / 2 c$. Eventually, in the static condition, the torque disappears and condition is true for tiny mass as well.

\section{References}

[1] Blanchet, L: Class.Quant.Grav.24, 3541(2007)

[2] Blanchet, L. and Tiec, A.: Phys.Rev.D80, 023524 (2009)

[3] D. Hajdukovic, Astrophysics and Space Science, 334, vol.2, 215 (2011)

[4] A. Raymond Penner, Astrophys. Space Sci. 361:124 (2016)

[5] E. Roza, doi: 10.20944/preprints201810.0713.v1(2018)

[6] E. Roza, doi: 1020944/preprints201705.0164.v4(2017)

[7] E. Roza, doi: 10.20944/preprints201712.0077.v3(2017)

[8] F. Kottler, Ann. Physik 56, 361, 401 (1918)

[9] E. Treffitz, Mathem. Ann. 86, 317 (1922)

[10] Li-Feng Sun et al., Modern Phys. Lett. A 28, 1350114 (2013)

[11] A. Einstein, Preuss. Akad. Wiss, Berlin (Math. Phys.), 142 (1917)

[12] www.scholarpedia.org/article/Cosmological constant

[13] J. Sola, Journal of Physics, Conf. Series, 453, 012015 (2013)

[14] P. Debye and E. Huckel, Physik. Zeitschrift, vol. 24, 9, 185 (1923) 
[15] E. Majorana, Nuovo Cimento, 14, 171 (1937)

[16] V. Mourik et al., Science, April 12, 2012; ArXiv: 1204.2792 (2012)

[17] D. Hestenes, arXiv: 0.802.2728v1 (2008)

[18] P.A.M. Dirac, Proc. Royal Soc. London, A 117, 610 (1928).

[19] C. Lanczos, F. Phys, 57, 447 (1929), reprint by W.R. Davis et al., North Car. St. Univ., Raleigh, Vol III, p2-1248 (1988)

[20] A.L. Fitzpartrick, K.M. Zurek, Phys. Rev.D 82, 075004 (2010)

[21] C.M. Ho, R.J. Scherrer, Phys. Lett. B 722, 341 (2013)

[22] Chun-Khiang Chua, Gwo-Guang Wong, arXiv:1512.01991v3 [hep-ph] (2016) 